\title{
Higher covariant derivative regulators and non-multiplicative renormalization
}

\author{
C.P. Martin ${ }^{\mathrm{a}}$, F. Ruiz Ruiz ${ }^{\mathrm{b}}$ \\ a Departamento de Física Teórica, C-XI, Universidad Autónoma de Madrid Cantoblanco, Madrid 28049, Spain \\ ${ }^{\mathrm{b}}$ NIKHEF-H, Postbus 41882, 1009 DB Amsterdam, The Netherlands \\ Received 31 October 1994 \\ Editor: P.V. Landshoff
}

\begin{abstract}
The renormalization algorithm based on regularization methods with two regulators is analyzed by means of explicit computations. We show in particular that regularization by higher covariant derivative terms can be complemented with dimensional regularization to obtain a consistent renormalized 4-dimensional Yang-Mills theory at the one-loop level. This shows that hybrid regularization methods can be applied not only to finite theories, like e.g. Chern-Simons, but also to divergent theories.
\end{abstract}

The renormalizability of a non-abelian gauge theory is not tied to the existence of a regularization method preserving its BRS symmetry, but to the more fundamental fact of the BRS symmetry not being anomalous [1]. Yet, having gauge invariant regularizations at our disposal is very useful for practical purposes. Sadly enough there are not many BRS invariant regularization methods available, dimensional regularization [2] and the method of higher covariant derivatives $[3,4]$ being the two most prominent ones.

Dimensional regularization is the most popular choice to regularize vector-like theories since the algebraic structure of these is not altered by a change in the dimension of spacetime. Ward identities hold almost automatically in this case. Unfortunately, when chiral objects like $\gamma_{5}$ matrices or Levi-Civita pseudotensors occur, an algebraically consistent dimensional regularization [5] is rather awkward, should it exist at all $[6,7]$. The reason for this is that the properties of chiral objects depend on the dimension of spacetime, which clashes somehow with the ideas behind dimensional regularization.

The method of higher covariant derivatives $[3,4]$ is a very interesting attempt to regularize non-abelian gauge theories while keeping the spacetime dimension at its physical value. Think for example of Yang-Mills theory. By adding suitable gauge invariant terms to the classical action - see Eq. (4) below - one transforms the thcory into a superrenormalizable gauge invariant theory, henceforth called higher covariant derivative theory. In this way partial regularization of Yang-Mills theory is achieved in a manifestly gauge invariant fashion. To regularize the few diagrams in the higher covariant derivative theory that are primitively divergent and at the same time preserve gauge invariance, one must supplement an additional gauge invariant regulator. Since these diagrams happen to be one-loop, finding a gauge invariant regulator that renders them finite should in principle be easier than addressing the regularization of the original Yang-Mills theory. The 
proposal in Ref. [4] for this additional regulator is a certain explicitly gauge invariant Pauli-Villars regularization whose latest version is explained in Ref. [8]. From now on, we will call higher covariant derivative Pauli-Villars regularization to this combination of higher covariant derivatives and Pauli-Villars regulators [8].

It has been recently shown [9] that the latter combination leads to a Yang-Mills theory with the wrong beta function. The reason being that there are nonlocal contributions to the vacuum polarization tensor that modify the non-local gauge-invariant part of the effective action. This no-go result renders higher covariant derivative Pauli-Villars regularization rather useless as a general regularization method. Nonetheless, since the anomalous contributions come from the Pauli-Villars determinants themselves [9], it might be possible that some other regularization of the higher covariant derivative theory led to a sensible Yang-Mills theory.

The purpose of this letter is to show that if we furnish the higher covariant derivative theory with dimensional regularization, one obtains a regularization method leading to a sensible 4-dimensional YangMills theory at the one-loop level. We will call this regularization method higher covariant derivative dimensional regularization and see that it is free of the problems that occur for higher covariant derivative Pauli-Villars regularization.

In this state of affairs, one may rightfully ask why not using dimensional regularization from the very beginning. In so doing we would save quite a lot of cumbersome algebra coming from the higher covariant derivative terms. In what follows we provide reasons to motivate the regularization method under consideration. Let us first consider perturbative three-dimensional Chern-Simons theory. The simplest higher covariant derivative term that one can add to the Chern-Simons action is $F^{2} / \Lambda$, where $\Lambda$ is a mass. This results into a very interesting theory on its own, namely topologically massive Yang-Mills theory, which when dimensionally regularized allows explicit two-loop computations. These computations have been performed in Ref. [7] and have shown the vanishing of the two-loop correction to the bare Chern-Simons parameter. They have also proved that topologically massive Yang-Mills theory is finite to all orders in perturbation theory, even though by power counting the theory is only superrenormalizable. With an eye on four-dimensional gauge theories involving $\gamma_{5}$ and the Levi-Civita symbol $\varepsilon^{\mu \nu \rho \sigma}$, it is therefore important to see whether the regularization method in question may become a general regularization procedure or it only works in three dimensions. Another reason is that if one shows that higher covariant derivative dimensional regularization is consistent, one then has an argument to not relinquish the possibility of a complete 4-dimensional regularization based on higher covariant derivatives. Last, but not least, is that by carrying on with this regularization method we shall learn how to renormalize theories whose divergences are parametrized by two regulators. This problem has not been tackled yet in a satisfactory way in the existing literature $[8,10]$ even at one loop.

Let us start by formulating higher covariant derivative dimensional regularization for 4-dimensional Yang-Mills theory. The classical Yang-Mills action for gauge group $S U(N)$ in a covariant gauge $\partial A^{a}=b^{a}$ is given by

$S=S_{\mathrm{YM}}+S_{\mathrm{GF}}$,

where

$$
\begin{aligned}
S_{\mathrm{YM}} & =\frac{1}{4} \int d^{4} x F_{\mu \nu}^{a} F^{a \mu \nu}, \\
S_{\mathrm{GF}} & =\int d^{4} x\left[\frac{\alpha}{2} b^{a} b^{a}-b^{a} \partial A^{a}\right. \\
+ & \left.\left(J^{a \mu}-\partial^{\mu} \bar{c}^{a}\right)\left(D_{\mu} c\right)^{a}-\frac{1}{2} f^{a b c} H^{a} c^{b} c^{c}\right] .
\end{aligned}
$$

The action $S$ is invariant under BRS transformations

$s A_{\mu}^{a}=\left(D_{\mu} c\right)^{a}, \quad s b^{a}=0, \quad s \bar{c}^{a}=b^{a}$,

$s c^{a}=-\frac{1}{2} g f^{a b c} c^{b} c^{c}, \quad s J_{\mu}^{a}=0, \quad s H^{a}=0$,

the BRS operator $s$ being nilpotent, $s^{2}=0$. We have introduced the external fields $J_{\mu}^{a}$ and $H^{a}$ to keep track of the renormalization properties of the composite operators $s A_{\mu}^{a}$ and $s c^{a}$, respectively. To achieve regularization of the ill-defined Feynman diagrams corresponding to the action $S$ in Eq. (1), one proceeds in two steps [11]. First, one introduces higher covariant derivative terms and replaces the classical gauge-fixed action $S$ with the action

$s_{\Lambda}=S_{\mathrm{YM}}+S_{2}+S_{\mathrm{GF}}^{f}$, 
where $S_{\mathrm{YM}}$ is as in Eq. (2) and $S_{2}$ and $S_{\mathrm{GF}}^{f}$ are given by

$$
\begin{aligned}
S_{2} & =\frac{1}{4 \Lambda^{4}} \int d^{4} x\left(D^{2} F_{\mu \nu}\right)^{a}\left(D^{2} F^{\mu \nu}\right)^{a}, \\
S_{\mathrm{GF}}^{f} & =\int d^{4} x\left[\frac{\alpha}{2} b^{a} \frac{1}{f^{2}\left(\partial^{2} / \Lambda^{2}\right)} b^{a}-b^{a} \partial A^{a}\right. \\
& \left.+\left(J^{a \mu}-\partial^{\mu} \bar{c}^{a}\right)\left(D_{\mu} c^{a}\right)-\frac{1}{2} f^{a b c} H^{a} c^{b} c^{c}\right] .
\end{aligned}
$$

Here $\alpha$ is the gauge fixing parameter and $f\left(\partial^{2} / \Lambda^{2}\right)$ is given in momentum space by

$f\left(\frac{p^{2}}{\Lambda^{2}}\right)=1+\frac{p^{4}}{\Lambda^{4}}$,

so as to ensure locality and render all $\alpha$-dependent contributions finite by power counting [9]. In our notation, $\alpha=0$ corresponds to the Landau gauge and $\alpha=1$ to the Feynman gauge. The action $S_{\Lambda}$ in Eq. (4) is invariant under the BRS transformations in Eq. (3) and gives rise to a supcrrenormalizable theory that we shall call higher covariant derivative theory. The free gauge propagator for this theory has the form

$$
\begin{aligned}
& G_{\mu \nu}^{a b}(p)=\delta^{a b} \frac{\Lambda^{4}}{p^{4}\left(p^{4}+\Lambda^{4}\right)} \\
& \quad \times\left[\left(p^{2} g_{\mu \nu}-p_{\mu} p_{\nu}\right)+\alpha \frac{\Lambda^{4}}{p^{4}+\Lambda^{4}} p_{\mu} p_{\nu}\right] .
\end{aligned}
$$

The ultraviolet behaviour of $G_{\mu \nu}^{a b}(p)$ is not strong enough as to make finite by power counting all 1PI Feynman diagrams. However, it is very easy to see that only one-loop 1PI diagrams contributing to the two-, three- and four-point Green functions of the gauge field are superficially divergent. In other words, the higher covariant derivative theory defined by $S_{\Lambda}$ is superrenormalizable. To regularize it, we use dimensionally regularization and thus achieve full regularization of the original Yang-Mills theory while preserving BRS invariance.

Having set a manifestly BRS invariant regularization method, however important this might be, is not the end of the story. Ultraviolet divergences have to be subtracted if a renormalized theory is to be obtained. In the case of gauge theories this means that given a regularization method, an explicitly BRS invariant subtraction procedure must be devised. Recall in this regard that dimensional regularization and ordinary abelian Pauli-Villars regularization lead to well established Bogoliubov-type subtraction algorithms $[5,12]$. To the best of our knowledge, no subtraction procedure has been shown to work for any higher covariant derivative regularization of non-abelian gauge theories. It would appear that setting such a procedure at one loop is a very simple task. After all, if a regularization method is BRS invariant, removing from the oneloop Green functions those contributions which are strictly UV divergent yields finite renormalized Green functions that verify the BRS identities at one loop. This being true, one must not forget that is not enough. General renormalization theorems in Lorentz covariant perturbation theory [13] imply that renormalized 1PI Green functions at one loop are unique modulo a polynomial in their momenta. Hence, if a regularization process modifies the non-local structure of the theory in the large cut-off limit, serious difficulties will arise. This is precisely what happens with higher covariant derivative Pauli-Villars regularization. In this case, renormalization at one loop can be accomplished in a BRS invariant manner and yet non-local gauge invariant radiative corrections get modified so as to yield an unphysical beta function [9]. Let us see that everything goes smoothly when higher covariant derivative dimensional regularization is used.

Let $\Phi$ collectively denote the fields $A_{\mu}^{a}, c^{a}, \bar{c}^{a}, b^{a}$, $J_{\mu}^{a}$ and $H^{a}$, and let $\epsilon$ denote the dimensional regulator defined by $D=4+2 \epsilon, D$ being the spacetime dimension. The regularized one-loop effective action $\Gamma_{1}(\Phi, \epsilon, \Lambda)$ obtained with the help of higher covariant derivative dimensional regularization depends on two regulators, $\epsilon$ and $\Lambda$. We want to fully characterize its UV divergent behaviour. To do this, we first report the one-loop values for the three 1PI functions that will allow us to do so. The vacuum polarization tensor $\Pi_{\mu \nu}^{a b}(p, \epsilon, \Lambda)$, the ghost self-energy $\Omega^{a b}(p, \epsilon, \Lambda)$ and ghost vertex $V_{\mu}^{a b}(k, p, \epsilon, \Lambda)$ as computed with higher covariant derivatives and dimensional regularization are given by

$$
\begin{aligned}
& \Pi_{\mu \nu}^{a b}(p, \epsilon, \Lambda)=-\delta^{a b}\left(p^{2} g_{\mu \nu}-p_{\mu} p_{\nu}\right) \\
& \quad \times\left(1+\frac{g^{2} c_{v}}{16 \pi^{2}}\left\{-\frac{71}{6}\left[\frac{1}{\epsilon}-\ln \left(\frac{\kappa^{2}}{\Lambda^{2}}\right)\right]\right.\right. \\
& \left.\left.\quad+\left(\frac{13}{6}-\frac{\alpha}{2}\right) \ln \left(\frac{p^{2}}{\Lambda^{2}}\right)+c_{0}(\alpha)\right\}\right)+\mathrm{vt},
\end{aligned}
$$




$$
\begin{aligned}
& \Omega^{a b}(p, \epsilon, \Lambda)=\left\{1+\frac{g^{2} c_{v}}{16 \pi^{2}}\left[\frac{3-\alpha}{4} \ln \left(\frac{p^{2}}{\Lambda^{2}}\right)\right.\right. \\
& \left.\left.+\omega_{0}(\alpha)\right]\right\} \delta^{a b} p^{2}+\mathrm{vt} \\
& V_{\mu}^{a b}(k, p, \epsilon, \Lambda) \\
& \quad=-i g f^{a b c}\left\{\left[1-\frac{g^{2} c_{V}}{16 \pi^{2}} \frac{\alpha}{2} \ln \left(\frac{p^{2}}{\Lambda^{2}}\right)\right] p_{\mu}\right. \\
& \left.+\frac{g^{2} c_{V}}{16 \pi^{2}} \alpha V_{\mu}^{\text {fin }}(k, p, \alpha)\right\}+\mathrm{vt} .
\end{aligned}
$$

Here "vt" stands for contributions that vanish when one takes the sequential limit $\epsilon \rightarrow 0, \Lambda \rightarrow \infty, \kappa$ is the dimensional regularization mass scale and $p^{\mu}$ and $k^{\mu}$ are external momenta. The symbols $c_{0}(\alpha)$ and $\omega_{0}(\alpha)$ denote constants which do not depend on $\kappa$ nor $\Lambda$. The function $V_{\mu}^{\text {fin }}(k, p, \alpha)$ is finite, has mass dimension 1 and does not depend on any of the dimensionful parameters $\kappa$ and $\Lambda$. A number of comments regarding these expressions are in order.

Comment 1. To obtain Eqs. (7)-(9), we have first performed a Laurent expansion around $\epsilon=0$, then dropped the terms that vanish as $\epsilon \rightarrow 0$ and finally discarded the terms that vanish as $\Lambda \rightarrow \infty$. To do all this we have used the techniques developed in Ref. [7]. Notice that this way to proceed implicitly assumes that to obtain renormalized 1 PI Green functions the regulators $\epsilon$ and $\Lambda$ should be removed in this order: first $\epsilon \rightarrow$ 0 and then $\Lambda \rightarrow \infty$. This prescription for removing the regulators is rather natural for the regularization method we are considering and the only well-defined one for Chern-Simons theory [7,14]. Proceeding the other way around, i.e. taking first $\Lambda \rightarrow \infty$ and then $\epsilon \rightarrow 0$, is rather messy and would eventually require renormalizing a theory at fixed but arbitrary $\epsilon$, something which is not clear how to achieve.

Comment 2. The vacuum polarization tensor in Eq. (7) has two types of UV singularities, a simple pole at $\epsilon=0$ and a logarithmic divergence in $\Lambda$. The pole comes from the fact that the higher covariant derivative theory has a divergent propagator at one loop. The singularity in $\Lambda$ stems from the divergent structure of Yang-Mills theory. Note in contrast that the ghost selfenergy and the ghost vertex do not show a singular behaviour at $\epsilon=0$. This is due to the the fact that for finite $\Lambda$ these two Green functions are convergent by power counting at $D=4$. As regards other Green functions, it is not difficult to see using general results for one-loop dimensionally regularized integrals [15] and the techniques in Ref. [7] that

(i) only the three and four-point Green functions for the gauge field will become singular at $\epsilon=0$, the singularity being a single pole, and

(ii) that any other superficially divergent one-loop IPI Green function will develop UV divergent singularities in $\ln \Lambda$.

Comment 3. Using the identity

$\ln \left(p^{2} / \Lambda^{2}\right)=\ln \left(p^{2} / M^{2}\right)+\ln \left(M^{2} / \Lambda^{2}\right)$,

where $M$ is a mass scale (later on to become the renormalization mass scale), we see that UV divergent contributions in the Green functions (7) - (9) are polynomial in the external momenta. Note that the non-local contributions explicitly displayed in Eqs. (7)-(9) are the same as those obtained with only dimensional regularization. Furthermore, the techniques in Ref. [7] ensure that also the non-local part in $V_{\mu}^{\text {fin }}(k, p, \alpha)$ in Eq. (9) is the same as for dimensional regularization, a fact that will be used later.

We now turn to the computation of the UV divergent contribution to the one-loop 1PI functional $\Gamma_{1}(\Phi, \epsilon, \Lambda)$. Recalling that the regularization method is explicitly BRS invariant and following standard techniques [16], we obtain that the one-loop effective action $\Gamma_{1}(\Phi, \epsilon, \Lambda)$ satisfies the BRS equation

$\Delta_{\Lambda, D} \Gamma_{1}(\Phi, \epsilon, \Lambda)=0$,

where $\Delta_{\Lambda, D}$ is the linearized Slavnov-Taylor operator for the $D$-dimensional counterpart of the action $S_{\Lambda}$ in Eq. (4). The operator $\Delta_{\Lambda, D}$ can be written as

$\Delta_{\Lambda, D}=\Delta_{D}+\int d^{\nu} \times \frac{\delta S_{2}^{D}}{\delta A_{\mu}^{a}} \frac{\delta}{\delta J^{a \mu}}$,

where $S_{2}^{D}$ is the $D$-dimensional version of the higher covariant derivative action in Eq. (5) and

$$
\begin{aligned}
\Delta_{D} & =\int d^{D} x\left[\frac{\delta S^{D}}{\delta A_{\mu}^{a}} \frac{\delta}{\delta J^{a \mu}}+\frac{\delta S^{D}}{\delta J_{\mu}^{a}} \frac{\delta}{\delta A^{a \mu}}\right. \\
+ & \left.\frac{\delta S^{D}}{\delta c^{a}} \frac{\delta}{\delta H^{a}}+\frac{\delta S^{D}}{\delta H^{a}} \frac{\delta}{\delta c^{a}}+b^{a} \frac{\delta S^{D}}{\delta \bar{c}^{a}}\right]
\end{aligned}
$$

is the Slavnov-Taylor operator for the gauge-fixed Yang-Mills action in $D$ dimensions $S^{D}$. In the limit $\epsilon \rightarrow 0, \Lambda \rightarrow \infty$ we may express $\Gamma_{1}(\Phi, \epsilon, \Lambda)$ as 
$\Gamma_{1}(\Phi, \epsilon, \Lambda)=\Gamma_{1}^{\text {div }}(\Phi, \epsilon, \Lambda)+\Gamma_{1}^{\mathrm{fin}}(\Phi)+$ "vt",

where again "vt" denotes contributions that vanish as $\epsilon \rightarrow 0, \Lambda \rightarrow \infty$. All finite contributions in this limit are collected in $\Gamma_{1}^{\text {fin }}(\Phi)$, whereas $\Gamma_{1}^{\text {div }}(\Phi, \epsilon, \Lambda)$ only contains contributions which are divergent. The functional $\Gamma_{1}^{\text {div }}(\Phi, \epsilon, \Lambda)$ is a four-dimensional integrated local functional of the fields and its derivatives with mass dimension 4 and ghost number 0 . Its coefficients are of the form $a_{1} \epsilon^{-1}+a_{2} \ln \Lambda+a_{3} \epsilon^{-1} \ln \Lambda$, with $a_{1}, a_{2}$ and $a_{3}$ constants. Using the methods in Ref. [7], it can actually be shown that $a_{3}=0$.

Let us look at Eq. (11). Since any one-loop 1PI diagram with at least one $J_{\mu}^{a}$ insertion is finite by power counting at $D=4, J$-dependent contributions to $\Gamma_{1}(\Phi, \epsilon, \Lambda)$ are finite as $\epsilon \rightarrow 0$. Recalling that all superficially divergent 1PI Green functions diverge as $\ln \Lambda$ for $\Lambda \rightarrow \infty$ and noting that the action $S_{2}$ has an overall factor $1 / \Lambda^{4}$, we have

$\lim _{\Lambda \rightarrow \infty} \lim _{\epsilon \rightarrow 0} \int d^{D} x \frac{\delta S_{2}^{D}}{\delta A_{\mu}^{a}} \frac{\delta \Gamma_{1}}{\delta J^{a \mu}}=0$.

Taking then the limit $\epsilon \rightarrow 0, \Lambda \rightarrow \infty$ in Eq. (11), it follows that $\Gamma_{1}^{\text {div }}(\Phi, \epsilon, \Lambda)$ satisfies

$\Delta \Gamma_{1}^{\mathrm{div}}(\Phi, \epsilon, \Lambda)=0$,

where $\Delta$ is the Slavnov-Taylor operator for the gaugefixed Yang-Mills action $S$ in 4 dimensions. The most general solution to this equation has long been known [16]; it has the form

$\Gamma_{1}^{\text {div }}(\Phi, \epsilon, \Lambda)=c_{1} S_{\mathrm{YM}}+\Delta X$,

where the functional $X$ is given by

$X=\int d^{4} x\left[c_{2}\left(J_{\mu}^{a}-\partial_{\mu} \bar{c}^{a}\right) A^{a \mu}+c_{3} H^{a} c^{a}\right]$

and $c_{1}, c_{2}$ and $c_{3}$ are coefficients. The coefficients $c_{1}, c_{2}$ and $c_{3}$ can be obtained from the singular contributions to three suitable 1PI Green functions. Choosing for these the vacuum polarization tensor, the ghost self-energy and the ghost vertex in Eqs. (7)-(9) and using the identity (10), we find that

$$
\begin{aligned}
& c_{1}=\frac{g^{2} c_{v}}{16 \pi^{2}}\left\{-\frac{71}{6}\left[\frac{1}{\epsilon}-\ln \left(\frac{\kappa^{2}}{\Lambda^{2}}\right)\right]+\frac{11}{3} \ln \left(\frac{M^{2}}{\Lambda^{2}}\right)\right\}, \\
& c_{2}=\frac{g^{2} c_{v}}{16 \pi^{2}} \frac{3+\alpha}{4} \ln \left(\frac{M^{2}}{\Lambda^{2}}\right),
\end{aligned}
$$

$c_{3}=\frac{g^{2} c_{V}}{16 \pi^{2}} \frac{\alpha}{2} \ln \left(\frac{M^{2}}{\Lambda^{2}}\right)$.

Putting everything together, we have that $\Gamma_{1}^{\text {div }}(\Phi, \epsilon, \Lambda)$ is given by Eqs. (12)-(13) with coefficients as in Eq. (14).

Once we have characterized the UV divergent behaviour of $\Gamma_{1}(\Phi, \epsilon, \Lambda)$, we are ready to define a renormalized BRS invariant 1 PI functional $\Gamma_{1}^{\mathrm{ren}}(\Phi, g, M)$ that describes Yang-Mills theory at the one-loop level. We define

$\Gamma_{1}^{\mathrm{ren}}(\Phi, g, M) \equiv \lim _{\Lambda \rightarrow \infty} \lim _{\boldsymbol{\epsilon} \rightarrow 0}(1-\mathbf{T}) \Gamma_{1}(\Phi, \epsilon, \Lambda)$,

where

$\mathbf{T} \Gamma_{1}(\Phi, \epsilon, \Lambda) \equiv\left(c_{1}+f_{1}\right) S_{\mathrm{YM}}^{D}+\Delta_{D} X^{D}$,

the functional $X^{D}$ reads

$$
\begin{aligned}
X^{D} & =\int d^{D} x\left[\left(c_{2}+f_{2}\right)\left(J_{\mu}^{a}-\partial_{\mu} \bar{c}^{a}\right) A^{a \mu}\right. \\
& \left.+\left(c_{3}+f_{3}\right) H^{a} c^{a}\right]
\end{aligned}
$$

and $f_{1}, f_{2}$ and $f_{3}$ are arbitrary $(\epsilon, \Lambda)$-independent constants. Note that Eqs. (12)-(14) guarantee that the limit in Eq. (15) is finite. Taking into account our considerations above, it is very easy to realize that the equations

$\Delta_{D} \mathrm{T \Gamma}_{1}(\Phi, \epsilon, \Lambda)=0$,

$\lim _{\Lambda \rightarrow \infty} \lim _{\epsilon \rightarrow 0} \int d^{D} x \frac{\delta S_{2}^{D}}{\delta A_{\mu}^{a}} \frac{\delta}{\delta J^{a \mu}} \mathbf{T} \Gamma_{1}(\Phi, \epsilon, \Lambda)=0$

hold, thus having

$\lim _{\Lambda \rightarrow \infty} \lim _{\epsilon \rightarrow 0} \Delta_{\Lambda, D} \Gamma_{1}^{\mathrm{ren}}(\Phi, g, M)=\Delta \Gamma_{1}^{\mathrm{ren}}(\Phi, g, M)$

and

$\Delta \Gamma_{1}^{\text {ren }}(\Phi, g, M)=0$.

In other words, the renormalized one-loop functional defined in Eq. (15) is BRS invariant. In fact, this is the most general 1PI functional that can be constructed from the regularized functional $\Gamma_{1}(\Phi, \epsilon, \Lambda)$ by performing local subtractions consistent with BRS invariance. This is due to the well-known fact $[1,16]$ that the most general finite renormalization compatible with 
BRS invariance amounts to adding to the renormalized 1PI functional the quantity $\int_{1} S+\Delta \int d^{4} x\left[f_{2}(J-\right.$ $\left.\partial \bar{c}) A+f_{3} H c\right]$, where $f_{1}, f_{2}$ and $f_{3}$ are arbitrary finite constants. But this is already taken into account in the definition of the Bogoliubov-type operator $\mathbf{T}$.

We now come to the computation of the one-loop beta function of the renormalized theory. It is plain that the renormalized 1PI functional cannot be obtained by multiplicative renormalizations of the coupling constant, the gauge parameter and the fields of the action $S_{\mathrm{A}}$ in Eq. (4). The reason is that such a multiplicative renormalization will give rise to new divergences at $\epsilon=0$ since the higher covariant derivative term $S_{2}$ will introduce divergent counterterms not associated with any primitively divergent diagram. Note that if we set $\alpha$ to zero and allow for a renormalization of $\Lambda$, or equivalently multiply $S_{2}$ by a bare parameter $\lambda_{0}$, our renormalized IPI functional can be obtained by multiplicative renormalization of a regularized bare theory having $S_{\Lambda}$ as classical action [10]. Strictly speaking, this renormalization procedure demands three renormalization conditions to define Yang-Mills theory, for one has to define what is meant by renormalized $\Lambda$, or equivalently renormalized $\lambda_{0}$. It is well-known [1] however that the construction of Yang-Mills theory along the lines of renormalized perturbation theory [13] requires only two renormalization conditions, as is the case with dimensional regularization. Hence, if we did renormalize $\Lambda$, we would have to understand Yang-Mills theory as the large- $\Lambda$ limit of a renormalized theory having $S_{\Lambda}$ as classical action. In this paper we are not concerned with this approach - it fails altogether for $\alpha \neq 0$ [10].

Having no multiplicative connection between the renormalized theory and a regularized bare theory, standard text book techniques cannot be used to compute beta functions and anomalous dimensions. Yet this should pose no problem, since the knowledge of the renormalized Green functions is all that is needed to fully characterize the theory. Let us recall in this regard that for the rigorously established BPHZ subtraction procedure there is no bare theory [1], the renormalized theory is obtained directly and everything, if liable of a perturbative calculation, can be computed from renormalized quantities. Since the beta function and the anomalous dimensions are the coefficients in the renormalization group equations for the renormal- ized Green functions, they can be determined from the latter equations. In our case the renormalization group equation for a 1 PI Green function $G_{R}\left(p_{e}, g, \alpha, M\right)$ reads ${ }^{\prime}$

$$
\begin{aligned}
& {\left[M \frac{\partial}{\partial M}+\beta(g) \frac{\partial}{\partial g}+\frac{1}{2} \sum_{\Phi} \gamma_{\Phi}(g) N_{\Phi}+\delta(g) \frac{\partial}{\partial \alpha}\right]} \\
& \times G_{R}\left(p_{e}, g, \alpha, M\right)=0 .
\end{aligned}
$$

The renormalized vacuum polarization tensor, ghost self-energy and ghost vertex in the minimal scheme $f_{1}=f_{2}=f_{3}=0$ are given by

$$
\begin{aligned}
& \Pi_{R_{\mu \nu}^{a b}}^{a b}(p)=-\left\{1+\frac{g^{2} c_{V}}{16 \pi^{2}}\left[\left(\frac{13}{6}-\frac{\alpha}{2}\right) \ln \left(\frac{p^{2}}{M^{2}}\right)\right.\right. \\
& \left.\left.+c_{0}(\alpha)\right]\right\} \delta^{a b}\left(p^{2} g_{\mu \nu}-p_{\mu} p_{\nu}\right), \\
& \Omega_{R}^{a b}(p)=\left\{1+\frac{g^{2} c_{\nu}}{16 \pi^{2}}\left[\frac{3-\alpha}{4} \ln \left(\frac{p^{2}}{M^{2}}\right)\right.\right. \\
& \left.\left.+\omega_{0}(\alpha)\right]\right\} \delta^{a b} p^{2}, \\
& V_{R}^{a b c}(k, p)=-i g f^{a b c}\left\{\left[1-\frac{g^{2} c_{V}}{16 \pi^{2}} \frac{\alpha}{2} \ln \left(\frac{p^{2}}{M^{2}}\right)\right] p_{\mu}\right. \\
& \left.+\frac{g^{2} c_{V}}{16 \pi^{2}} \alpha V_{\mu}^{\mathrm{fin}}(k, p, \alpha)\right\} .
\end{aligned}
$$

Substituting these expressions in Eq. (16) and expanding in powers of $g$ the functions $\beta(g), \gamma_{\Phi}(g)$ and $\delta(g)$, one obtains a linear system to be verified by the coefficients of such series. Solving this system leads to

$$
\begin{aligned}
& \beta(g)=-\frac{11}{3} \frac{g^{3} c_{v}}{16 \pi^{2}}+O\left(g^{4}\right) \\
& \gamma_{A}(g)=\left(\frac{13}{3}-\alpha\right) \frac{g^{2} c_{v}}{16 \pi^{2}}+O\left(g^{4}\right) \\
& \gamma_{c}(g)+\gamma_{\bar{c}}(g)=(3-\alpha) \frac{g^{2} c_{V}}{16 \pi^{2}}+O\left(g^{4}\right) \\
& \delta(g)=O\left(g^{2}\right)
\end{aligned}
$$

These results are in complete agreement with the beta function and the anomalous dimensions computed

\footnotetext{
${ }^{1}$ Note that the renormalized 1 PI functional $\Gamma_{1}^{\mathrm{ren}}(\Phi, g, M)$ is defined by a Bogoliubov-type subtraction from a regularized 1PI functional compatible with a quantum action principle. This ensures that the renormalization group equation holds true for the renormalized Green functions.
} 
within any consistent mass independent renormalization schemc known as yet, say the MS scheme for dimensional regularization.

Let us make two final remarks. First, note that the non-local part of the renormalized Green functions in Eqs. (17) are the same as for any renormalization scheme based on dimensional regularization. This, together with the fact that the renormalized 1PI functional can be constructed from the three Green functions in Eqs. (17), implies that the renormalized effective action $\Gamma_{1}^{\text {ren }}(\Phi, g, M)$ we have constructed here and the one-loop dimensionally renormalized 1PI functional differ by finite renormalizations of the fields and the parameters in the theory. Our second remark concerns the existence to all orders in perturbation theory of a consistent BRS invariant renormalization algorithm based on higher covariant derivative dimensional regularization. Although this problem lies well outside the scope of this paper, let us say that the subtraction procedure should take care of the singularities at $\epsilon=0$ first and then remove the divergent large- $\Lambda$ behaviour. The BRS invariance of the algorithm should come from BRS invariance of the regularization method and the fact the the purely divergent UV contributions are BRS invariant on their own. Although we do not have a rigorous proof of these statements, we have partially checked them for two-loop diagrams with overlapping divergences.

F.R.R. was supported by FOM, The Netherlands. The authors also acknowledge partial support from CICyT, Spain.

\section{References}

[1] O. Piguet and A. Rouet, Phys. Rep. 76 (1981) 1.

[2] G. 't Hooft and V. Veltman, Nucl. Phys. B 44 (1972) 189

[3] A.A. Slavnov, Nucl. Phys. B 31 (1971) 301; B.W. Lee and J. Zinn-Justin, Phys. Rev. D 5 (1972) 3121

[4] A.A. Slavnov, Theor- Math. Phys. 33 (1977) 977.

[5] P. Breitenlohner and D. Maison, Commun. Math. Phys. 52 (1977) 11

[6] C.P. Martin, Phys. Let. B 241 (1990) 513.

[7] G. Giavarini, C.P. Martin and F. Ruiz Ruiz, Nucl. Phys. B 381 (1992) 222.

[8] L.D. Faddeev and A.A. Slavnov, Gauge Fields, Introduction to Quantum Theory, second edition (Benjamin, Reading 1990).

[9] C.P. Martin and F. Ruiz Ruiz, Higher covariant derivative Pauli-Villars regularization does not lead to a consistent QCD, FTUAM 94/9, NIKHEF-H 94/24.

[10] M. Day, Nucl. Phys. B 231 (1983) 1.

[11] A.A. Slavnov, Symmetry preserving regularization for gauge and supergauge theories, in: Superspace and Supergravity, edited by S.W. Hawking and M. Rocek, p. 177 (Cambridge University Press, Cambridge 1981)

[12] N.N. Bogoliubov and D.V. Shirkov, Introduction to the Theory of Quantized Fields (Wiley-Interscience, New York).

[13] H. Epstein and V. Glaser, Ann. Inst. Henri Poincaré 29 (1973) 211.

[14] G. Leibbrandt and C.P. Martin, Nucl. Phys. B 377 (1992) 593 ;

G. Giavarini, C.P. Martin and F. Ruiz Ruiz, Phys. Lett. B 314 (1993) 324:

G. Leibbrandt and C.P. Martin, Nucl. Phys. B 416 (1994) 351 ;

G. Giavarini, C.P. Martin and F. Ruiz Ruiz, Phys. Lett. B 332 (1994) 345.

[15] J.C. Collins, Renormalization (Cambridge University Press, Cambridge, 1987).

[16] C. Itzykson and J-B Zuber, Quantum Field Theory (McGraw-Hill Book Company). 